BULLETIN OF THE

AMERICAN MATHEMATICAL SOCIETY

Volume 78, Number 4, July 1972

\title{
LATTICES OF INVARIANT SUBSPACES
}

\author{
BY WILLIAM ARVESON ${ }^{1}$
}

Communicated by Irving Glicksberg, December 27, 1971

Let $\mathscr{A}$ be an algebra of operators on a Hilbert space $\mathscr{H}$, and let Lat $\mathscr{A}$ denote the lattice of all $\mathscr{A}$-invariant subspaces. Recall [4] that $\mathscr{A}$ is said to be reflexive if every operator which leaves invariant every element of Lat $\mathscr{A}$ is already in $\mathscr{A}$. Thus, reflexive operator algebras are completely determined by their invariant subspace lattices, and one might hope to get useful information about the algebra $\mathscr{A}$ by studying the lattice Lat $\mathscr{A}$. One question that arises here is, which specific lattices occur as the lattice Lat $\mathscr{A}$ of some algebra $\mathscr{A}$ ? Such lattices are called reflexive (it is very easy to see that reflexive lattices have an equivalent definition which is dual to the definition of reflexive algebra). In this paper we announce some results of a study of reflexive lattices; full details will appear elsewhere.

Before going further, we want to describe the type of concrete problems which led to these considerations. To specify a reflexive algebra $\mathscr{A}$ on a separable space $\mathscr{H}$, it is equivalent to specify a sequence $\mathfrak{M}_{1}, \mathfrak{M}_{2}, \ldots$ of closed subspaces of $\mathscr{H}\left(\mathscr{A}\right.$ is then defined as $\left.\left\{T: T \mathfrak{M}_{i} \subseteq \mathfrak{M}_{i}, i \geqq 1\right\}\right)$. To get a fairly general class of examples, let $n$ be a positive integer, and let $X$ be the countable Cartesian product $X_{1} \times X_{2} \times \cdots$ of the sets $X_{i}$ $=\{1,2, \ldots, n\} . X$ comes equipped with a product Borel structure, and we define subsets $E_{i j} \subseteq X, i \geqq 1,1 \leqq j \leqq n$, to be the cylinders

$$
E_{i j}=\left\{x \in X: x_{i} \leqq j\right\},
$$

where $x_{i}$ denotes the $i$ th coordinate of the sequence $x$. Now choose a finite measure $\mu$ on $X$, let $\mathscr{H}=L^{2}(X, \mu)$, and define subspaces $\mathfrak{M}_{i j}$ of $\mathscr{H}$ by $\mathfrak{M}_{i j}=L^{2}\left(E_{i j}, \mu\right)$. Then the operator algebra

$$
\mathscr{A}(X, \mu)=\left\{T \in \mathscr{L}(\mathscr{H}): T \mathfrak{M}_{i j} \subseteq \mathfrak{M}_{i j} \text {, for all } i, j\right\}
$$

is reflexive, and it always contains the multiplication algebra of $L^{\infty}(X, \mu)$. Of course, the properties of these algebras will depend strongly on the measure $\mu$. We want to take up two types of special cases:

Problem 1. Let $n \geqq 2$, and define $\mu$ on $X$ by $\mu=\prod_{1}^{\infty} \mu_{i}$, where each $\mu_{i}$ assigns uniform mass $1 / n$ to each point of $\{1,2, \ldots, n\}$. Let $\mathscr{A}_{n}$ be the associated operator algebra. Can $\mathscr{A}_{m}$ and $\mathscr{A}_{n}$ be similar if $m \neq n$ ?

Problem 2. Take $n=2$, and choose a number $p, 0<p<1$. Let $\mu$ $=\prod_{1}^{\infty} \mu_{i}$, where each $\mu_{i}$ assigns mass $p$ to $\{1\}$ and mass $1-p$ to $\{2\}$. Let $\mathscr{A}_{p}$ be the associated operator algebra. Can $\mathscr{A}_{p}$ and $\mathscr{A}_{q}$ be similar if $p \neq q$ ?

AMS 1970 subject classifications. Primary 41A15; Secondary 46L15.

1 Research supported by NSF grant GP-5585. 
As we will see, these two problems illustrate different phenomena, and the answers to both are no. The solution involves two steps. First, one shows that the complete lattice $\mathscr{L}(X, \mu)$ generated by the subspaces $\mathfrak{M}_{i j}$ is always reflexive (this follows from a general result, Theorem 2 below). It follows that $\mathscr{L}(X, \mu)=\operatorname{Lat} \mathscr{A}(X, \mu)$, so to show that $\mathscr{A}(X, \mu)$ and $\mathscr{A}(Y, v)$ are not similar, it suffices to show that their invariant subspace lattices are not isomorphic. This second part of the analysis derives from the results of $\S \S 2$ and 3.

1. Reflexive lattices. By a subspace lattice we mean a sublattice of the lattice of all selfadjoint projections of some Hilbert space (it is more convenient here to speak of projections rather than subspaces), which contains 0 and 1 , and is closed in the strong operator topology. It is easy to see that subspace lattices are complete as lattices, and every reflexive lattice is a subspace lattice [4]. Of course, there are simple examples of subspace lattices which are not reflexive.

It is known that if a subspace lattice is linearly ordered ([6], [7]), or is isomorphic to an atomic Boolean algebra [5], then it is reflexive. In this section we discuss separably-acting commutative subspace lattices $\mathscr{L}$ (i.e. $P Q=Q P$ for all $P, Q$ in $\mathscr{L}$ ), subject to no other side conditions. We begin by describing a general construction. By a partially ordered Borel space we mean a partially ordered set $(X, \leqq)$ endowed with a sigma-field of subsets of $X ;(X, \leqq)$ is called standard if $X$ is standard as a Borel space [1], and there exists a sequence $f_{n}$ of real-valued Borel functions on $X$ such that $x \leqq y$ iff $f_{n}(x) \leqq f_{n}(y)$ for every $n \geqq 1$. As an example, one may take $X$ to be the Cartesian product $X_{1} \times X_{2} \times \cdots$ where each $X_{i}$ is the finite set $\{1,2, \ldots, n\}$ (for a fixed value of $n$ ). The Borel structure is of course the product structure, and the partial order is defined by $x \leqq y$ iff $x_{i} \leqq y_{i}$ for every $i$, where $x_{i}$ denotes the $i$ th coordinate of the sequence $x$. A Borel set $E$ is called decreasing if whenever $x \in E$ and $y \leqq x$, then $y \in E$. The family of all decreasing Borel sets forms a sigma-lattice of subsets of $X$ which is usually not closed under complementation.

Finally, let $\mu$ be a finite measure on $X$. Then each decreasing Borel set $E$ determines a projection $P_{E}$ in $L(X, \mu)$, and the family $\mathscr{L}(X, \leqq, \mu)$ of all such projections turns out to be a complete sublattice of the projection lattice in $L^{\infty}(X, \mu)$. By causing the elements of $\mathscr{L}(X, \leqq, \mu)$ to act in the usual way on the Hilbert space $L^{2}(X, \mu)$ we obtain an example of a commutative subspace lattice. Significantly, this construction exhausts all of the (separable) possibilities:

THEOREM 1. Every separably-acting commutative subspace lattice is isomorphic to the lattice $\mathscr{L}(X, \leqq, \mu)$ of some standard partially ordered measure space. 
We remark that there are a number of variants of Theorem 1, one of which concludes that the isomorphism is implemented by a unitary equivalence (one simply has to take appropriate account of higher multiplicities). Thus, the study of commutative subspace lattices is reduced to the study of the lattices $\mathscr{L}(X, \leqq, \mu)$.

We now state the main result of this section.

THEOREM 2. Every separably-acting commutative subspace lattice is reflexive.

Theorem 2 depends on the following rather technical result which, as it turns out, seems to lie at the heart of many of the natural questions about reflexive lattices as well as reflexive operator algebras.

THEOREM 3. Let $X$ and $Y$ be compact metric spaces, let $\mu$ and $v$ be sigmafinite Borel measures on $X$ and $Y$ respectively, and let $K$ be a closed subset of $X \times Y$. Assume that $\sigma(K)=0$ for every finite positive measure $\sigma$ on $X \times Y$ satisfying $\sigma_{1} \ll \mu$ and $\sigma_{2} \ll \nu$, where $\sigma_{1}(E)=\sigma(E \times Y)$ and $\sigma_{2}(F)=\sigma(X \times F)$. Then there exist Borel sets $M \subseteq X$ and $N \subseteq Y$ such that $\mu(M)=v(N)=0$ and $K \subseteq M \times Y \cup X \times N$.

2. The structure of certain lattices. As we pointed out in the introduction, classification problems for reflexive operator algebras largely reduce to classification problems for their invariant subspace lattices. In this section we shall consider a general class of lattices which is tailored to these questions. Throughout, $L$ will denote a distributive complete metric lattice [3]; that is, a distributive lattice $L$, having a 0 and a 1 , and which admits a positive isotone valuation $v: L \rightarrow[0,+\infty)$, with the property that $L$ is a complete metric space relative to the distance function $d(x, y)$ $=v(x \vee y)-v(x \wedge y)$. Thus, $L$ is the lattice-theoretic counterpart of a (finite) measure algebra, the principal difference being that in the lattice case one may not be able to take complements. One should keep in mind the examples $\mathscr{L}(X, \leqq, \mu)$ of the preceding section; here, the valuation $v$ is defined on the projection $P_{E}, E \subseteq X$, by $v\left(P_{E}\right)=\mu(E)$.

If $\left\{L_{n}\right\}$ is any family of sublattices of $L, \vee_{n} L_{n}$ will stand for the complete sublattice generated by $\bigcup_{n} L_{n}$. By a factorization for $L$ we mean a sequence $L_{1}, L_{2}, \ldots$ of finite sublattices of $L$, each containing the 0 and 1 of $L$, such that

(i) $L=\vee_{n} L_{n}, L_{n} \neq\{0,1\}$,

(ii) (zero-one law) $\bigcap_{n=1}^{\infty}\left(L_{n} \vee L_{n+1} \vee \cdots\right)=\{0,1\}$,

(iii) (independence) for all $a, b$ in $L_{n}$ and all $x, y$ in $\vee_{i \neq n} L_{i}, a \wedge x \leqq b$ $\vee y$ implies $a \leqq b$ or $x \leqq y$.

This will be expressed by the notation $L=\bigotimes_{n} L_{n}$. A factorization $L=\bigotimes_{n} L_{n}$ is called indecomposable if each $L_{n}$ is indecomposable in the sense that, for each $n$, the only factorizations of $L_{n}=A \otimes B$ are when $A=L_{n}$ or $B=L_{n}$. 
As a special case, let $L$ be the separable nonatomic measure algebra. Then it is easy to see that $L$ admits a great variety of indecomposable factorizations which are in no way related to one another. Thus it may be surprising that, if $L$ is at the opposite extreme from a Boolean algebra, then unique factorization does hold. The relevant definition is the following: $L$ is called primary if for any two of its elements $x, y, x \vee y=1$ implies $x=1$ or $y=1$.

THEOREM 4 (Unique Factorization Theorem). Let $L$ be a primary distributive complete metric lattice. Then for any two indecomposable factorizations $L=\bigotimes_{n} L_{n}=\bigotimes_{n} L_{n}^{\prime}$, there is a permutation $\pi$ of the positive integers such that $L_{n}^{\prime}=L_{\pi(n)}$.

To apply this result to Problem 1 , let $\mathscr{L}_{n}$ (for $n$ fixed) be the complete lattice generated by the given projections of Problem 1 . Then it follows easily from Theorem 4 that $\mathscr{L}_{m}$ and $\mathscr{L}_{n}$ are not isomorphic if $m \neq n$. Utilizing Theorem 2 it can be shown that $\mathscr{L}_{n}=$ Lat $\mathscr{A}_{n}$, and thus we conclude that $\mathscr{A}_{m}$ and $\mathscr{A}_{n}$ are not similar if $m \neq n$.

Note, however, that Theorem 4 does not distinguish between the lattices of Problem 2. The next section deals with that type of problem.

3. Numerical invariants. We now want to introduce a numerical invariant which will distinguish between the lattices involved in Problem 2. The reader may find the following discussion to be reminiscent of the Kolmogorov-Sinai invariant of ergodic theory [2].

Let $L$ be an infinite complete distributive metric lattice, which is separable as a metric space. By a generator for $L$ we mean a countable subset $\left\{x_{1}, x_{2}, \ldots\right\}=G$ of $L$ which generates $L$ as a complete lattice (we shall also require that the labelling satisfy $x_{m} \neq x_{n}$ if $m \neq n$ ). A state of $L$ is defined as an isotone valuation $\mu$ (not necessarily related to the distinguished valuation giving rise to the metric on $L$ ), such that $\mu(0)=0$ and $\mu(1)=1 ; \mu$ is called normal if for every increasing sequence $x_{n} \in L$ one has $\sup _{n} \mu\left(x_{n}\right)=\mu\left(\vee_{n} x_{n}\right)$, and dually for decreasing sequences. For a given generator $G=\left\{x_{1}, x_{2}, \ldots\right\}$ and a given normal state $\mu$ we define

$$
\alpha(G, \mu)=\lim _{n} \inf \mu\left(x_{n}\right) .
$$

It is easy to see that $\alpha(G, \mu)$ does not depend on the particular labelling of the elements of $G$. Thus,

$$
\alpha(L)=\inf _{G, \mu} \alpha(G, \mu),
$$

the inf taken over all generators $G$ and all normal states $\mu$, is a numerical isomorphism invariant of the lattice $L$, which takes values in the unit interval. We now describe a procedure for computing $\alpha(L)$ in quite a wide variety of cases. A prime in a distributive lattice $L$ is an element $p$ of 
$L$ such that $x \vee y=p$ implies $x=p$ or $y=p . \mathscr{P}(L)$ will denote the set of all nonzero primes of $L$. Note that if $L$ is the lattice of all positive integers (partially ordered by $x \leqq y$ iff $x$ divides $y$ ), then the primes are the elements of the form $p^{n}$ where $p$ is an ordinary prime and $n \geqq 1$. If $L$ is a finite distributive lattice then it is generated by $\mathscr{P}(L)$; indeed every nonzero element is a union of primes [3]. The main result of this section is the following.

THEOREM 5. Let $L$ be an infinite distributive complete metric lattice which has a factorization $L=\bigotimes_{n} L_{n}$ into primary lattices $L_{n}$. Let $P=\bigcup_{n=1} \mathscr{P}\left(L_{n}\right)$, and let $\mu$ be any normal state of $L$. Then $P$ is a generator, and moreover

$$
\alpha(L)=\alpha(P, \mu) .
$$

Theorem 5 allows one to compute $\alpha\left(\mathscr{L}_{p}\right)$ where $\mathscr{L}_{p}, 0<p<1$, is the complete lattice generated by the sequence of subspaces given in Problem 2. It turns out that $\alpha\left(\mathscr{L}_{p}\right)=p$, so that $\mathscr{L}_{p}$ and $\mathscr{L}_{q}$ are not isomorphic if $p \neq q$. An argument similar to the one given in the preceding section now shows that the operator algebras $\mathscr{A}_{p}$ and $\mathscr{A}_{q}$ are not similar if $p \neq q$.

We also remark that the techniques of $\S 1$ can be applied to the dual problem of determining when an operator algebra is reflexive. This aspect too will be discussed elsewhere.

\section{REFERENCES}

1. W. B. Arveson, Representations of $C^{*}$-algebras, Studies in Operator Theory (to appear).

2. P. Billingsley, Ergodic theory and information, Wiley, New York, 1965. MR 33 \# 254.

3. G. Birkhoff, Lattice theory, 2 nd rev. ed., Amer. Math. Soc. Colloq. Publ., vol.' 25 , Amer. Math. Soc., Providence, R.I., 1948. MR 10,673.

4. P. R. Halmos, Ten problems in Hilbert space, Bull. Amer. Math. Soc. 76 (1971), 887-933. MR 42 \# 5066.

5. - Reflexire lattices of subspaces (to appear).

6. R. V. Kadison and I. M. Singer, Triangular operator algebras. Fundamentals and hyper-reducible theory, Amer. J. Math. 82 (1960), 227-259. MR 22 \#12409.

7. J. R. Ringrose, On some algebras of operators, Proc. London Math. Soc. (3) 15 (1965), 61-83. MR 30 \# 1405.

Department of Mathematics, University of CALIFornia, Berkeley, CAlifornia 94720 